\title{
MedienPädagogik
}

Themenheft Nr. 27: Tagungsband: Spannungsfelder und blinde Flecken. Medienpädagogik zwischen Emanzipationsanspruch und Diskursvermeidung. Herausgegeben von Sven Kommer, Thorsten Junge und Christiane Rust.

\section{Editorial zum Tagungsband: \\ Spannungsfelder und blinde Flecken. Medienpädagogik zwischen Emanzipationsanspruch und Diskursvermeidung}

\author{
Sven Kommer, Thorsten Junge und Christiane Rust
}

Die erziehungswissenschaftliche Auseinandersetzung mit Medien hat in Deutschland eine lange und vielfältige Tradition. In den letzten Jahrzehnten hat sich die Medienpädagogik zu einer eigenständigen akademischen (Teil-)Disziplin entwickelt, die in der (Fach-)Öffentlichkeit wahrgenommen wird und sich aktiv in aktuelle gesellschaftliche Diskurse einbringt. Sichtbar wird die Etablierung als Fach u. a. daran, dass an einer Reihe von Hochschulen Professuren geschaffen wurden, die für medienpädagogische Studienelemente oder -gänge und/oder Zusatzausbildungen verantwortlich sind. Der gestiegene Stellenwert der Disziplin zeigt sich auch an ihrer Rolle in der Deutschen Gesellschaft für Erziehungswissenschaft, innerhalb derer sie sich von einer «Arbeitsgemeinschaft auf Zeit» (Mitte der 1990erJahre) zu einer eigenständigen Sektion (seit 2010) entwickelt hat.

Angesichts der gegenwärtig zu beobachtenden Entwicklungen im Medienbereich (u. a. Digitalisierung, Globalisierung, Algorithmisierung), deren Folgen für gesellschaftliche und pädagogische Prozesse und Bereiche kaum zu unterschätzen sind, erstaunt es, dass die Medienpädagogik bis heute dazu neigt, Rechtfertigungsstrategien für die eigenen Existenz zu generieren. Bei einer kritischen Betrachtung (z. B. der universitären Stellenpolitik) ist aber zu konstatieren, dass sich die Medienpädagogik auch 40 Jahre nach dem Erscheinen von Dieter Baackes wegweisender Schrift Kommunikation und Kompetenz ihrer Anerkennung und festen Verankerung im akademischen Feld tatsächlich noch immer nicht sicher sein kann.

Eine Ursache hierfür liegt möglicherweise in der noch immer grossen (und oftmals geradezu zelebrierten) Heterogenität des Faches. So sind die unterschiedlichen Traditionslinien des pädagogischen Umgangs mit Medien, die mitunter nur bedingt miteinander vereinbar sind, bis heute zu beobachten und wirkmächtig. Zu unterscheiden ist hierbei bspw. eine auf Emanzipation, kommunikative Kompetenz und Selbstermächtigung orientierte Linie, die sich in ausserschulischen Settings subjekt- und ressourcenorientiert die Medien als Instrumente subversiver Strategien und/oder der Identitätskonstruktion angeeignet hat. Oft nur bedingt an diese anschlussfähig ist eine schulpädagogische Linie, die ihren Ausgang bei der «Filmund Medienerziehung' genommen hat und inzwischen stärker auf Kompetenzerwerb fokussiert. Daneben findet sich dann noch eine (vielfältig ausdifferenzierte) «Mediendidaktik», die immer wieder technologie-euphorisch innovatives Lernen versprochen hat. 
Positiv gewendet, zeigt bereits diese kleine Skizze, dass die Medienpädagogik als erziehungswissenschaftliche Disziplin darauf verweisen kann, wie intensiv Theoriebildung, Forschung und Praxis gegenwärtig in einem breiten Spektrum von Ansätzen, Forschungsschwerpunkten und «Schulen〉 vollzogen wird. Das Fach ist also sehr lebendig - erscheint aber aus der Perspektive externer Beobachter möglicherweise auch als diffus. Dies zeigt sich nicht zuletzt im Text von Edwin Keiner, mit dem wir unseren Tagungsband abschliessen werden (siehe unten). Vor diesem Hintergrund erscheint eine Bilanzierung und Reflexion hilfreich, um die eigene Verortung und Ausrichtung einer kritisch-konstruktiven Überprüfung zu unterziehen. Denn ein selbstkritischer Blick auf Gegenwart und Geschichte der Medienpädagogik offenbart neben vielfältigen innovativen Erträgen auch blinde Flecken, Diskursvermeidungen und (möglicherweise ideologisch bedingte) Vereinseitigungen. Die im Raum stehenden Fragen und Reflexionsbedarfe gemeinsam im Rahmen einer Sektionstagung zu bearbeiten erschien der Sektion Medienpädagogik gewinnbringend, sodass die Frühjahrstagung 2015 in Aachen (19. und 20. März 2015) unter dem Titel «Spannungsfelder und blinde Flecken. Medienpädagogik zwischen Emanzipationsanspruch und Diskursvermeidung» ausgerichtet wurde.

Die Anlage der Tagung zielte darauf, Raum für eine konstruktive Auseinandersetzung und eine produktive Selbst-Irritation zu schaffen. Die widersprüchlichen Tendenzen «Emanzipation` (insbesondere als Zielgrösse medienpädagogischer Arbeit) und ¿Diskursvermeidung) (u. a. bei aufkommendem Verdacht allzu bewahrpädagogischer Positionen) sowie - teilweise direkt damit verbundene - Fragen zu Subversion und Affirmation sollten und konnten intensiv diskutiert werden. Zur Strukturierung des Feldes wurden vom Tagungsteam im Vorfeld drei zentrale Spannungsfelder herausgearbeitet, die sowohl im Call wie auch bei der Gestaltung des Programms als Strukturierungsgrundlage dienten:

1. Medienpädagogik und die Grenzen von Subjektorientierung und Kulturalismus.

2. Medienpädagogik und informatorische Bildung.

3. Medienpädagogik als Akteur in kapitalistischen, kommerzialisierten und globalisierten Medienwelten.

Bereits im Verlauf der Tagung wurde von mehreren Seiten der Wunsch geäussert, die Vorträge und Diskurse in Form eines Tagungsbands zu verschriftlichen und so zugänglich zu machen. Als Organisationsteam haben wir diesen Wunsch gerne realisiert. Mit Klaus Rummler (geschäftsführender Herausgeber der Zeitschrift MedienPädagogik; www.medienpaed.com) hat die Sektion Medienpädagogik dabei einen wertvollen Partner gewonnen, sodass wir unseren Tagungsband nun als online frei zugängliche Publikation präsentieren können. Die freie Verfügbarkeit der unterschiedlichen Beiträge entspricht dabei auch unserem aktuellen Verständnis von Open Access und Open Educational Resources. 
An dieser Stelle möchten wir es als Herausgeber-Team nicht versäumen, einigen Personen explizit für ihre Unterstützung zu danken. Der Vorstand der Sektion im Frühjahr 2015, bestehend aus Petra Grell, Theo Hug und Johannes Fromme, hat die Sektionstagung in bewährter Weise unterstützt und stand dem Tagungs-Team hilfsbereit zur Seite. Die kurzen Kommunikationswege und das grosse Interesse an unserem Tagungsthema erleichterten uns die Vorbereitung und ermöglichten es uns, unsere Konferenz in der geplanten Weise umzusetzen.

Ohne das unermüdliche Engagement unserer Mitarbeiterin Corinna Haas im Vorfeld und während der Tagung, wäre das Treffen der Sektion nicht in dieser Form möglich gewesen. Sie hat sich in herausragender Weise um alle Fragen gekümmert, die im Vorfeld und während einer solchen Tagung zu klären sind.

\section{Zu den Beiträgen}

Der Tagungsband beginnt mit einem Beitrag von Horst Niesyto (PH Ludwigsburg), der mit seinem Vortrag den zweiten Veranstaltungstag eröffnet hatte. Im Mittelpunkt von «Medienpädagogik und digitaler Kapitalismus» steht die These von der Medienkritik als zentraler Aufgabe der Medienpädagogik. Ausgangspunkt der Überlegungen ist die Wahrnehmung, dass die Medienpädagogik als Disziplin bei der Auseinandersetzung mit und der Partizipation an einer kritischen Medien- und Gesellschaftsanalyse noch Nachholbedarf hat. Die besondere gesellschaftliche Verantwortung der Medienpädagogik begründet Horst Niesyto ausgehend von der gegenwärtigen Entwicklung in der digitalen Medienlandschaft, die mit dem Stichwort «digitaler Kapitalismus» umschrieben wird.

\section{Spannungsfeld 1: Medienpädagogik und die Grenzen von Subjektorientierung} und Kulturalismus

In dem der Tagung vorausgehenden Call for Papers ${ }^{1}$ war dieses Spannungsfeld wie folgt bestimmt:

Die wissenschaftlichen Diskurse der Medienpädagogik [wie sie sich in der Sektion der DGfE und der GMK konstituiert] sind über weite Strecken von der Abwehr «bewahrpädagogischer» Positionen und deren Externalisierung in Maßnahmen des Jugendmedienschutzes geprägt. Der ursprüngliche kritische Impetus hat dabei an Relevanz verloren. Vor dem Hintergrund der ¿Cultural Studies», der erziehungswissenschaftlichen «Alltagswende», einer weitreichenden Subjekt- und Ressourcenorientierung und nicht zuletzt der popkulturellen Unterstellung von Subversion stellt sich die Frage, ob

1 https://blog.rwth-aachen.de/fruehjahrstagung-der-sektion-medienpaedagogik/files/2014/09/Callfor-Papers-09252014_final.pdf. 
medienpädagogische Forschung und Theoriebildung nicht streckenweise zu einer mehr oder weniger unreflektierten Affirmation des je aktuellen medienkulturellen und/oder medientechnischen Hypes regrediert ist. Und damit aus dem Blick verliert, dass sich möglicherweise auch «kontraproduktive» und sproblematisches Nutzungsformen und Inhalte beobachten lassen.

Diskussionswürdig erscheinen in diesem Kontext auch der Umgang mit dem Feld der ehemaligen Hochkultur, die Positionierung zu den Anforderungen des Bildungssystems und die Auseinandersetzung mit Prozessen sozialer Ungleichheit.

Pointierter formuliert: Ist hier eine medienpädagogische «Déformation professionelles zu beobachten, die dazu führt, dass aus der Angst, als Kulturpessimistln gelabelt zu werden, geradezu reflexhaft jegliche mediale Artikulation und Nutzungsform überbewertet und bei öffentlicher Kritik verteidigt wird?

Den Einstieg in dieses Feld markiert der Beitrag «Das Potential der Medien für die Bildung des Subjekts» von Rudolf Kammerl (inzwischen FAU Erlangen). Er widmet sich vorrangig der Frage, ob und inwieweit die Strukturen internetbasierter Kommunikation die Subjektwerdung erschweren können. Vor dem Hintergrund der Annahme, dass eine Subjektkonzeption stets auch einer reflexiven Selbstvergewisserung bedarf, greift Kammerl verschiedene theoretische Zugriffe auf und diskutiert das Verhältnis von Medien und Subjekt. Das Tagungsmotto aufgreifend wird dabei auch geprüft, welche Blickverengungen und -erweiterungen medienpädagogischer Theoriebildung und Forschung in diesem Forschungsfeld wahrzunehmen sind.

Ebenfalls in diesem Feld angesiedelt ist der Beitrag «Medien - Diskurs - Kritik Potenziale der Diskursforschung für die Medienpädagogik» von Valentin Dander (Universität zu Köln). Hier wird der blinde Fleck der Medienpädagogik entlang einer vielschichtigen Analyse des Diskurses im Bereich der Medienpädagogik betrachtet. Dabei wird sowohl der Diskurs an und für sich, als auch die Bereiche des Subjektes und der Machtverhältnisse thematisiert und anschliessend auf die aktuelle Diskursforschung in der Medienpädagogik angewendet.

Einen «Blick über den Tellerrand` fordert der Beitrag «Medienpädagogik als Kulturwissenschaft!? - Überlegungen zu disziplinären Öffnungen und Anschlüssen» von Patrick Bettinger (Universität Hamburg). Er diskutiert die Möglichkeiten, die sich durch eine verstärkte Einbringung kulturwissenschaftlicher Perspektiven in die Medienpädagogik eröffnen können. Besondere Aufmerksamkeit erfährt an dieser Stelle der Kulturbegriff, der von verschiedenen Seiten beleuchtet wird. Bettinger zielt letztlich darauf ab, die kulturwissenschaftlichen Anschlussmöglichkeiten der Medienpädagogik mit Blick auf neue theoretische wie empirische Perspektiven 
aufzuzeigen. Der Autor arbeitet heraus, wie hierdurch Lücken im medienpädagogischen Fachdiskurs geschlossen werden könnten.

Der letzte der im ersten Spannungsfeld angesiedelten Beiträge trägt den Titel «Blinde Flecken und präskriptive Unschuld. Zur Morphologie medienpädagogischer Urteilsformen» und stammt von Rainer Leschke (Universität Siegen). Aus einer medienwissenschaftlichen Perspektive unterzieht Rainer Leschke die Medienpädagogik einer kritischen Revision. Dabei legt er gewissermassen den Finger in die Wunde, wenn er bspw. die medienpädagogischen Reaktionsmuster im Kontext jeweils neuer Medienentwicklungen genauer betrachtet und dabei sich wiederholende Reaktions- und Bearbeitungsmuster findet. Als Fazit unterstellt Rainer Leschke der Medienpädagogik ein unzureichend bearbeitetes Reflexionsdefizit. Der Rechtfertigungsstress, dem unsere Disziplin bisweilen gegenübersteht, wird dabei pointiert erörtert.

Ausgangspunkt von Wolfgang Ruges (Universität Wien) Beitrag «Disziplinlose Pluralität» ist die Feststellung, dass eine zufriedenstellende Wissenschaftstheorie der Medienpädagogik bis dato nicht vorliegt. Er geht diesem Umstand nach, indem er die (z.T. stark abweichende) institutionelle Anbindung der Medienpädagogik an Universitäten, die unterschiedlichen Konfigurationen des Gegenstandes sowie die Legitimationsargumentationen hinsichtlich der Notwendigkeit von Medienpädagogik betrachtet. Der (konstatierte) Mangel an Homogenität führt ihn u. a. zu der Frage, ob man nicht lieber von Medienpädagogiken im Plural sprechen sollte.

\section{Spannungsfeld 2: Medienpädagogik und informatische Bildung}

Der Call ${ }^{2}$ formuliert hier bereits eine Perspektive, die im Jahr 2016 u. a. mit der Veröffentlichung der «Dagstuhl-Erklärung: Bildung in der digitalen vernetzten Welt» nochmals an Relevanz gewinnt:

Der NSA-Skandal und die Datensammelwut der digitalen Dienstleister machen deutlich, wie sehr die aktuellen Medienwelten von Strukturen der automatisierten Informationsverarbeitung durchdrungen sind. Die Auseinandersetzung mit den Strukturen, Prozessen und Techniken unter den bunten Oberflächen ist bisher aber nur wenig entwickelt. Mit Blick auf die Intentionen von Medienkompetenz/Medienbildung ist diese Lücke hoch problematisch, sind hier doch weitreichende Folgen sowohl auf gesellschaftlicher wie auch individueller Ebene denkbar (oder bereits realisiert). Zu fragen ist, ob nicht gerade hier eine wichtige Aufgabe darin bestünde, gemeinsam mit anderen Disziplinen proaktiv mitzugestalten.

\footnotetext{
2 https://blog.rwth-aachen.de/fruehjahrstagung-der-sektion-medienpaedagogik/files/2014/09/Callfor-Papers-09252014_final.pdf.
} 
Im Rahmen der Tagung gingen die verschiedenen Beiträge u. a. der Frage nach, wie in diesem Bereich eine zukünftige Verortung der Medienpädagogik auszusehen hat (z. B. im Kontext Hochschule) und mit welchen zukünftigen Herausforderungen die Profession zu rechnen hat.

So wenden sich Nina Grünberger (Universität Wien) und Stephan Münte-Goussar (Europa-Universität Flensburg) zunächst dem Bildungsort Schule zu. In ihrem Beitrag « «Medienbildung in der Schule〉 oder 〈Schule im Medium»» erörtern sie vor dem Hintergrund einer zunehmenden Mediatisierung der kindlichen Lebenswelt die damit verbundenen Implikationen für die Schulentwicklung. Die (notwendige) Entwicklung von Schule wird dabei als Transformationsprozess der Schulkultur «im Mediums beschrieben, der nicht bei der Implementierung von medialen Tools in den weitgehend gleich bleibenden Unterricht stehen bleiben darf.

Mit dem Bereich der «Soziale Arbeit» fokussiert Christian Helbig (Technische Hochschule Köln) ein Handlungsfeld, welches im medienpädagogischen Diskurs häufig wenig Beachtung findet. In «Die Mediatisierung professionellen Handelns. Zur Notwendigkeit von Handlungskompetenzen im Kontext digitaler Medien in der Sozialen Arbeit» erörtert er, welche Auswirkungen die Etablierung digitaler online-Medien für die Arbeit der Fachkräfte im Bereich der Sozialen Arbeit hat. Angesichts der Tatsache, dass digitale Medien zunehmend den Alltag der Adressaten/-innen als auch die Institutionen und Organisationen Sozialer Arbeit durchdringen, eröffnen sich neue Handlungsfelder aber auch neue individuelle Herausforderungen. Im Kontext der Professionalisierung der Sozialen Arbeit erlangt somit auch die Vermittlung von (kritischer) Medienkompetenz und medienpädagogischer Kompetenz eine neue Bedeutung.

Mandy Schiefner-Rohs (Technische Universität Kaiserslautern) fokussiert in ihrem Beitrag, wie es in der Medienpädagogik durchaus typisch ist, auf die Diskussion um digitale Medien in der Schule. Aber sie erweitert den Blickwinkel, indem sie die Auswirkungen einer Medialität für menschliche Kommunikations- und Bildungsprozesse und die (z. T. hieraus resultierenden) Folgen einer Kultur der Digitalität für die Schule diskutiert. Sie kann u. a. fundiert darlegen, dass sich die medienpädagogische Forschung stärker der Rekonstruktion von medialen Handlungspraktiken der Schülerinnen und Schüler sowie der Lehrkräfte widmen sollte.

Spannungsfeld 3: Medienpädagogik als Akteur in kapitalistischen, kommerzialisierten und globalisierten Medienwelten

Im Call wurde die Stossrichtung dieses dritten Feldes sehr deutlich formuliert: Die Diskussionen um die Problematiken von «big data haben eindrücklich vor Augen geführt, dass gerade auch die Welt der digitalen, vernetzten und globalisierten Medien längst der Logik einer turbokapitalistischen Gewinnmaximierung 
unterworfen sind. Nach dem Verschwinden der 'Kritischen Theorie» und ihrer Auseinandersetzung mit der 'Kulturindustries findet sich im Mainstream der akademischen Medienpädagogik nur selten eine theoretisch und empirisch grundierte Auseinandersetzung mit den Folgen der immer rasanteren Kommerzialisierung, Marketing-Formierung und Zielgruppenorientierung der Medienanbieter. Vor dem Hintergrund der aktuellen Entwicklungen erscheint diese Tendenz zur Diskursvermeidung als ein hoch problematischer blinder Fleck - dessen Bearbeitung aber weder durch einen Rückfall in allzu plumpe Medienschelte semi-frankfurter Provenienz noch durch Verklärung des Prosumerverhaltens angeraten scheint.

Die Beiträge zu diesem Themenkreis widmen sich Fragen nach Bedingungen, Chancen und Grenzen der Medienpädagogik in kommerzialisierten Welten.

Dabei werden Spotlights auf die Disziplin Medienpädagogik und deren Handlungsfelder geworfen. Zunächst erörtert Alessandro Barberi (Universität Wien) ob und warum «Medienpädagogik als Sozialtechnologie» zu verstehen ist. Ausgehend von Dieter Baackes Habilitationsschrift Kommunikation und Kompetenz, die für die Genese der Medienpädagogik von zentraler Bedeutung war (und ist), werden die Forschungsergebnisse eines Close Readings von Baackes Diskursbegründung präsentiert. Barberi zielt darauf ab, dessen Theoriebildung diskursanalytisch und wissenssoziologisch im Kontext der zeitgeschichtlichen Entstehung zu analysieren. Es wird u. a. aufgezeigt, dass die gegenwärtig geführten Debatten zur «Medienkompetenz» schon bei Baacke archäologisch mit der Marxschen Philosophie der Praxis verbunden waren. Vor diesem Hintergrund wird vorgeschlagen, Baackes Theoriebildung über Habermas und Bourdieu im Sinne einer praxeologischen und handlungsorientierten Medienpädagogik zu verbinden.

Die Frage einer möglichen Diskursvermeidung wird im Aufsatz «Warum Computerspielen trotzdem gut ist - Neutralisierungsstrategien von Computerspielabhängigen und sozialwissenschaftlichen Forschern» von Paula Bleckmann und Nadine Jukschat deutlich. Mit «Computerspielabhängigkeit» greifen die Autorinnen ein Thema auf, welches nicht nur innerhalb der Medienpädagogik kontrovers diskutiert wird. Die Verfasserinnen stellen dar, welche Neutralisierungsstrategien von Computerspielerlnnen aus ihrer Sicht angewendet werden und wie diese - kommuniziert durch die medienpädagogische Forschung - Eingang in den gegenwärtigen Diskurs finden.

In ihrem Beitrag «Medienpädagogische Aufgabenfelder hinsichtlich der Visualität im digitalen Zeitalter» erörtert Petra Missomelius welche Aufgaben die Medienpädagogik in den (neuen) visuellen Medienkulturen zu bewältigen hat. Dies wird vor allem im Hinblick auf die Unterschiede zum Umgang mit den traditionellen Bildmedien herausgearbeitet.

Den Abschluss dieses Themenblocks bildet der Beitrag von Johannes Fromme (Otto-von-Guericke-Universität Magdeburg). Er widmet sich in «Medienpädagogen/ 
innen auf dem Arbeitsmarkt - Ergebnisse einer Verbleibsstudie» den beruflichen Handlungsfeldern von Absolvent/innen, die einen medienpädagogischen Studiengang belegt haben. Somit wird eine Frage aufgegriffen, die für den (erfolgreichen) Fortbestand unserer Disziplin ausserhalb des Bildungsortes Schule überaus grosse Relevanz besitzt.

Der Tagungsband schliesst mit einer Verschriftlichung des Eröffnungsvortrags von Edwin Keiner ab. In seinem Beitrag «Didaktik - Bildung - Technik - Kritik. Medienpädagogik und Antinomien der Moderne» unterzieht er die Medienpädagogik in einem Rückblick auf die vergangenen Jahrzehnte einer überaus kritischen Betrachtung. Er vollzieht dies anhand der Analyse der Problemfelder «Disziplinäre Autonomie», «Interdisziplinarität» sowie «Internationalität» und stellt jeweils die Schwierigkeiten und Defizite der Medienpädagogik heraus. Unter Bezugnahme auf die Leitbegriffen «Bildung», «Didaktik», «Technik» und "Kritik» skizziert Keiner abschliessend Anschlussmöglichkeiten für die Medienpädagogik und verschiedene Übersetzungsmöglichkeiten in einen interdisziplinären und internationalen Raum.

Mit den vorliegenden Texten dokumentieren wir die Erträge der Frühjahrstagung in Aachen. Auch wenn damit ein überaus grosses Spektrum an Themen und Fragestellungen abgedeckt wird, sind wir uns sehr wohl bewusst, dass auch dieses Themenheft seine «blinden Flecken» aufweist. Er ist somit als spannendes Element eines ebenso spannenden Diskurses/Feldes zu verstehen. Die gegenwärtigen und zukünftigen Entwicklungen im pädagogischen und im Medienbereich werden dafür sorgen, dass sich die Medienpädagogik auch in den nächsten Jahrzehnten ein hoch relevantes und von herausfordernden und nicht selten spannenden Fragen geprägtes Feld bleiben wird. Und wir werden sehen, wann das nächste Mal der Zeitpunkt für eine kritische Selbstbetrachtung gekommen ist, um blinde Flecken, neue Spannungsfelder und Ansätze der Diskursvermeidung zu erörtern sind.

Aachen, Oktober 2017: Sven Kommer, Thorsten Junge und Christiane Rust 